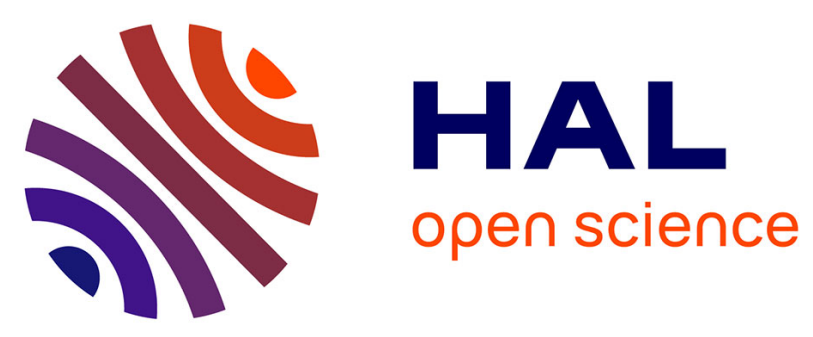

\title{
Noise measurement and modeling of a dual-frequency VECSEL at cesium clock wavelength (Oral)
}

\author{
Liu Hui, Fabienne Goldfarb, Nessrine Guerchi, Chang-Hoong Chow, Fabien
}

Bretenaker, Ghaya Baili, Paul Dumont, Gaëlle Lucas-Leclin, Isabelle Sagnes, Gregoire Beaudoin

\section{To cite this version:}

Liu Hui, Fabienne Goldfarb, Nessrine Guerchi, Chang-Hoong Chow, Fabien Bretenaker, et al.. Noise measurement and modeling of a dual-frequency VECSEL at cesium clock wavelength (Oral). 2017 International Topical Meeting on Microwave Photonics (MWP), Oct 2017, Beijing, China. pp.Th1.9, 10.1109/MWP.2017.8168649 . hal-01782308

\section{HAL Id: hal-01782308 \\ https://hal-iogs.archives-ouvertes.fr/hal-01782308}

Submitted on 3 Mar 2019

HAL is a multi-disciplinary open access archive for the deposit and dissemination of scientific research documents, whether they are published or not. The documents may come from teaching and research institutions in France or abroad, or from public or private research centers.
L'archive ouverte pluridisciplinaire HAL, est destinée au dépôt et à la diffusion de documents scientifiques de niveau recherche, publiés ou non, émanant des établissements d'enseignement et de recherche français ou étrangers, des laboratoires publics ou privés. 


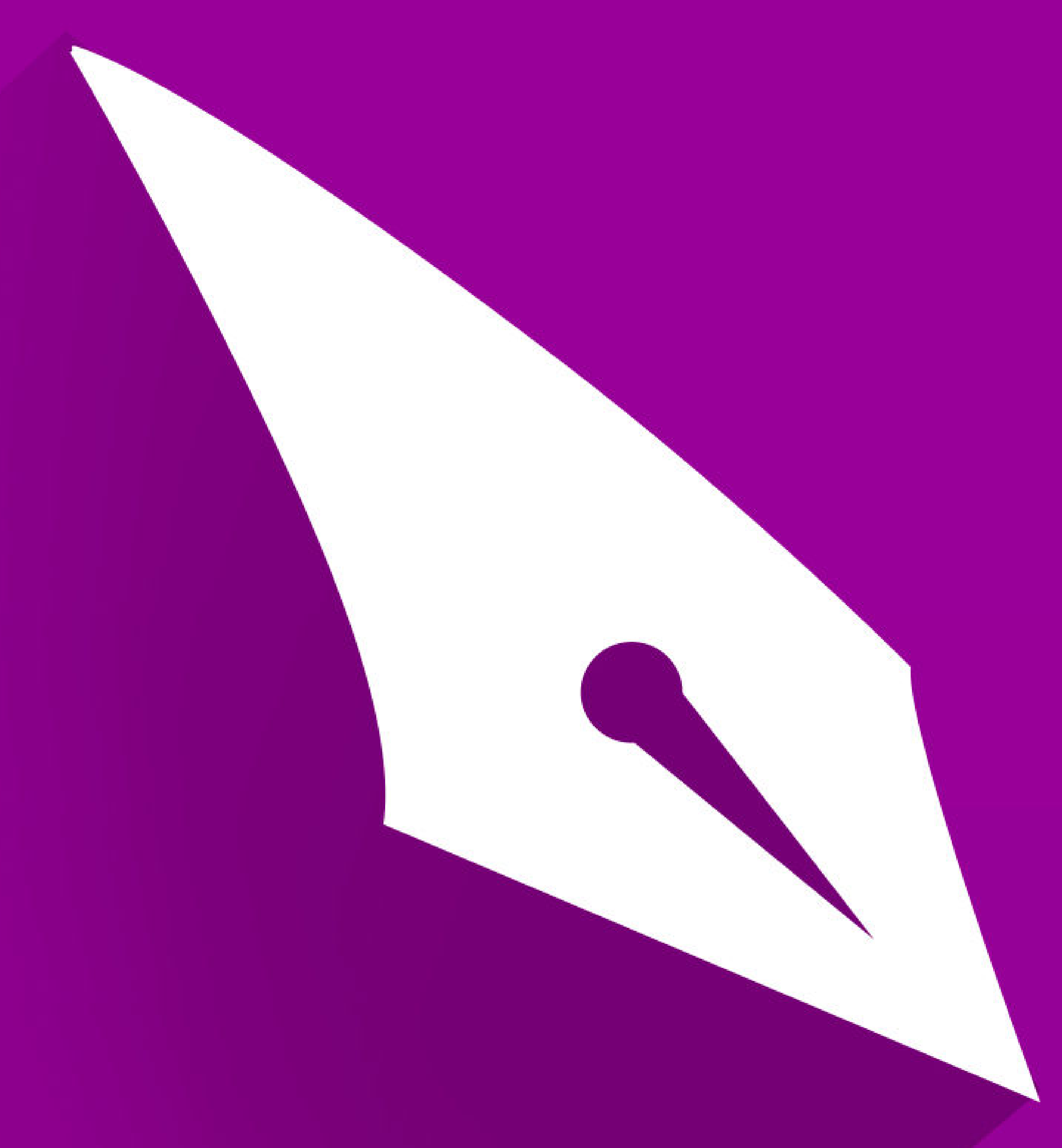

\section{福昕PDF编辑器 个人版 \\ - 永久・轻巧・自由}

\section{立即下载}

\section{购买会员}

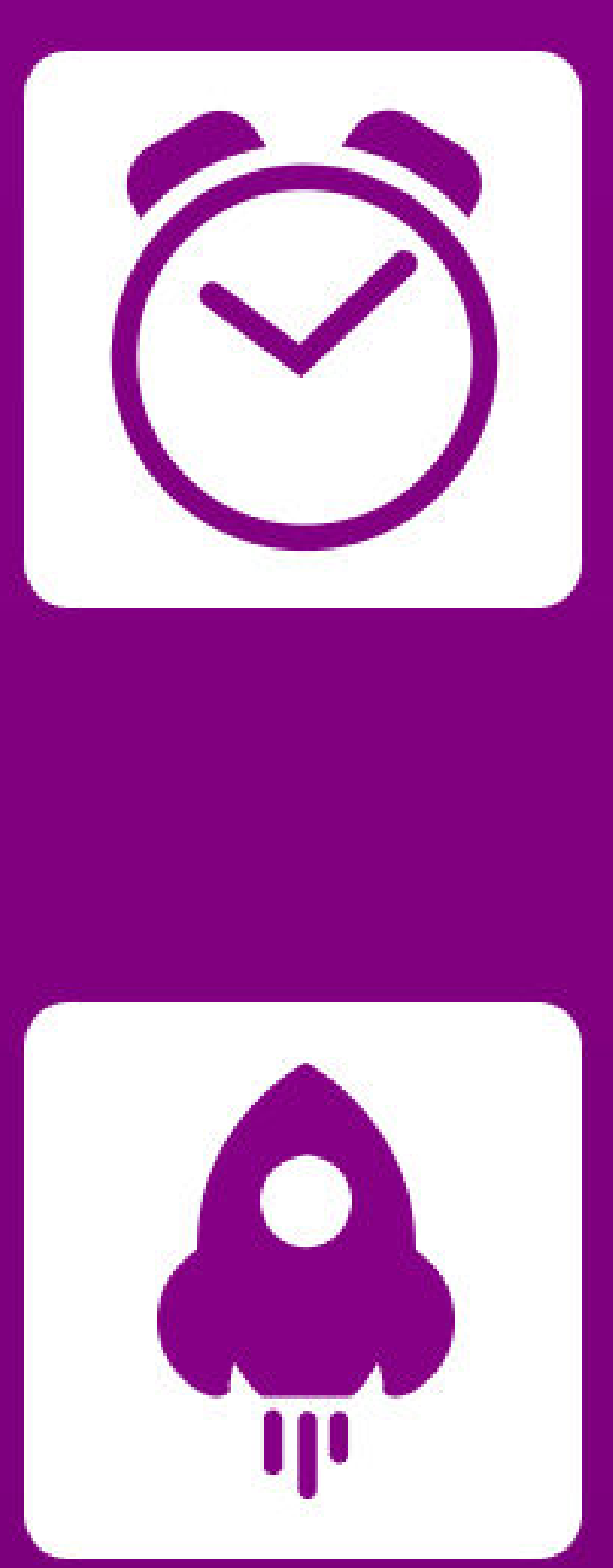

永久使用

无限制使用次数

\section{极速轻巧}

超低资源占用，告别卡顿慢

\section{自由编辑}

享受Word一样的编辑自由


○扫一扫，关注公众号 


\section{Noise measurement and modeling of a dual-frequency VECSEL at cesium clock wavelength}

\author{
Hui Liu, Fabienne Goldfarb, Nessrine Guerchi, Chang- \\ Hoong Chow, and Fabien Bretenaker \\ Laboratoire Aimé Cotton, CNRS - Université Paris Sud - \\ ENS Paris-Saclay, Université Paris-Saclay \\ Orsay, France \\ hui.liu@u-psud.fr \\ Ghaya Baili \\ Thales Research \& Technology \\ Palaiseau, France
}

\author{
Paul Dumont, Gaëlle Lucas-Leclin \\ Laboratoire Charles Fabry \\ CNRS - Institut d'Optique Graduate School \\ Orsay, France \\ Isabelle Sagnes, Grégoire Beaudoin \\ Centre de Nanosciences et de Nanotechnologies, CNRS - \\ Université. Paris-Sud - Université Paris-Saclay - C2N \\ Marcoussis \\ Marcoussis, France
}

\begin{abstract}
We study the noise of a class-A dual-frequency vertical external cavity surface emitting laser at Cesium clock wavelength. Both the intensity noise and phase noise of the beatnote between the two orthogonal polarized modes are measured and modeled. The intensity noise of the two orthogonal laser modes and its correlation are well predicted by a theory based on modified rate equations. The phase noise of the beatnote is predicted by the theory based on thermal effect and phase/amplitude coupling effect.
\end{abstract}

Keywords-Dual-frequency laser; vertical external cavity surface emitting laser; intensity noise; phase noise.

\section{INTRODUCTION}

The generation of optically carried radio frequency (RF) is an important issue in the area of microwave photonics (MWP), which has gained tremendous developments and has a bright future [1]. Dual-frequency vertical-external-cavity surfaceemitting laser (DF-VECSEL) [2], sustaining two crosspolarized laser in a same optical cavity, is an attractive solution to generate such an optically carried RF signal. With class-A dynamics, DF-VECSEL can exhibit a low intensity noise and be free of relaxation oscillation (RO) [3], due to the fact that the lifetime of the photons inside the external cavity can be longer than the lifetime of the semiconductor gain media carriers. Additionally, DF-VECSEL is also interesting for atomic clocks. Indeed, coherent population trapping (CPT) atomic clock [4] is promising to realize miniaturized atomic clocks, but requires a trade-off between performance and size. Recently, a report gives out a solution using two pulses and a double lambda atomic system to obtain high contrast Ramsey fringes [5]. It is expected that the trade-off can be improved by the combination of DF-VESCEL and the double lambda atomic system configuration of CPT atomic clocks [6].

The study of noise of the DF-VECSEL is important and interesting, because it conveys information on the laser dynamics, enabling us to understand and control its behavior. DF-VECSEL was first experimentally demonstrated at $1 \mu \mathrm{m}$ [2], showing the advantage of no RO, which is inherent to Class-B laser, for example, Nd:YAG [7]. Later, the DFVECSEL at telecom wavelength $(1.55 \mu \mathrm{m})$ [8] was reported and its behavior in terms of spectra of intensity and phase noises was investigated in theory and experiment [9][10]. The experimental results were very well predicted by a theory based on modified rate equations. The validity of this theory has been even more solid after the success of the analysis of the correlation between the different noises [11]. Rich information about the laser can be revealed using this theory. Meanwhile, a DF-VECSEL specifically designed for Cesium CPT atomic clock was reported [6]. Since the modified rate equations successfully applied to the telecom wavelength DF-VECSEL, it is worth applying this theory to another DF-VECSEL with different wavelength to validate its generality.

This paper reports a DF-VECSEL wavelength at cesium clock. A special attention is paid in the measurement and modeling of the intensity noise and phase noise. The modeling is based on the modified rate equations.

\section{CHARACTERISTICS OF THE DF-VECSEL}

\section{A. Configuration of the DF-VECSEL}

Figure 1 is a schematics of the DF-VECSEL. The cavity is composed of a semiconductor chip the temperature of which is stabilized at $20{ }^{\circ} \mathrm{C}$ by a Peltier cooler and a heat sink, and a concave mirror with a $5 \mathrm{~cm}$ radius of curvature. The transmittivity of concave mirror is $0.5 \%$ at the laser wavelength $(852 \mathrm{~nm})$. A $\mathrm{YVO}_{4}$ birefringent crystal with a thickness of $0.5 \mathrm{~mm}$, cut at $45^{\circ}$ to its optical axis anti-reflection coated, is inserted inside the cavity to create two optical paths, also introducing a spatial separation. The transverse separation between the two orthogonally separated laser modes, denoted as $d$, is about $50 \mu \mathrm{m}$. The etalon, with a thickness of $150 \mu \mathrm{m}$, is used for making each of the cross-polarized modes to emit in a single longitudinal mode. The length of the cavity is $48.0 \mathrm{~mm}$, corresponding a laser mode with a waist of $50 \mu \mathrm{m}$ on the semiconductor chip.

The semiconductor chip is composed of a Bragg mirror and an active structure. The Bragg mirror contains 32.5 pairs of half-wavelength $\mathrm{AlAs} / \mathrm{Al}_{0.22} \mathrm{GaAs}$ layers. The reflectivity of this Bragg mirror is very close to $100 \%$. The active layers contain seven quantum wells (8nm-GaAs) embedded in 
$\mathrm{Al}_{0.22} \mathrm{GaAs}$ barriers, which absorb the pump laser, two layers of $30 \mathrm{~nm} \mathrm{Al}_{0.39} \mathrm{GaAs}$ used for the carrier confinement, and protective layers $(50 \mathrm{~nm} \mathrm{InGaP}$ and $5 \mathrm{~nm}$ GaAs) to prevent aluminum oxidation. All of the quantum wells are designed to be located at antinodes of the laser field. When a pump beam with spot diameter of $80 \mu \mathrm{m}$ is incident on the chip with $40^{\circ}$ incident angle, the reflected power is $25 \%$, indicating that the absorption the pump power is about $75 \%$. This chip is designed without anti-reflection coating to increase the gain of the mode resonant with the micro-cavity created in the semiconductor structure.

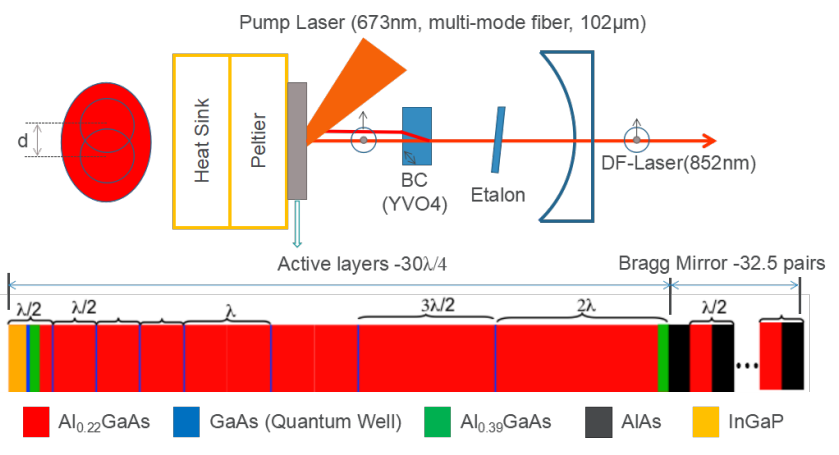

Fig. 1. The schematic of the DF-VECSEL

The pump laser, at a wavelength of $673 \mathrm{~nm}$, is a laser diode coupled to a multimode fiber with a $102 \mu \mathrm{m}$ core diameter. The incident angle of the pump laser is $40^{\circ}$, leading to a $100 \mu \mathrm{m} \times$ $130 \mu \mathrm{m}$ elliptical pump spot on the semiconductor chip. For this experiment, the pump power is set at 1.1 Watt.

\section{B. Observation of two mode oscillation and beatnote}

The two mode oscillation was monitored by observing the transmission signal of a Fabry - Perot interferometer (FPI), which is shown in Fig. 2(a). The beatnote was analyzed by a electric spectrum analyzer (ESA). Due to the $1 \mathrm{GHz}$ limitation of response bandwidth of the photo-detector (PD) and 500 $\mathrm{MHz}$ bandwidth of the radio-frequency amplifier (RFA), the frequency of the beatnote was adjusted to be equal to $350 \mathrm{MHz}$. As shown in Fig.2(b), above the floor, a noise pedestal can be seen at the foot of the beatnote peak, with a few tens of $\mathrm{MHz}$ bandwidth, which will be discussed in the next section.
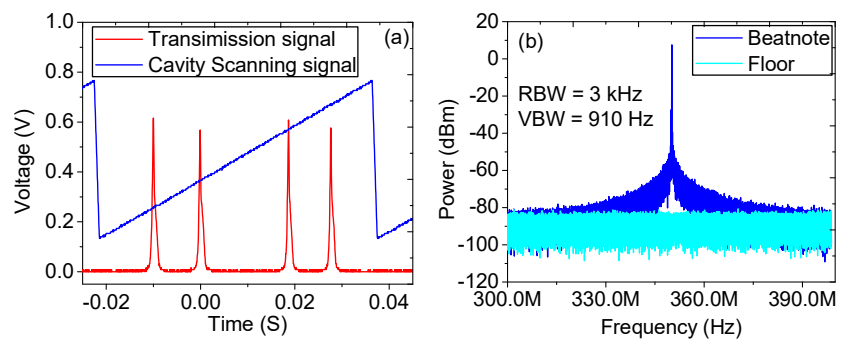

Fig. 2. (a) Optical spectrum of the DF-VECSEL analyzed by FPI. (b) Beatnote spectrum analyzed by ESA

\section{NOISE MEASUREMENT AND MODELING}

\section{A. Pump noise measurement}

Since both the intensity noise of the two orthogonally polarized modes and the phase noise of the beatnote mainly come from the pump noise, it is necessary to characterize this pump noise. The pump laser is multimode fiber coupled, so the modal noise exists in the pump beam due to the varying interference between the numerous spatial modes. The pump noise that eventually feeds the laser intensity and phase noises must be considered only in the regions of the semiconductor chip that amplify the two VECSEL laser modes. This fact requires us to extract the pump noises just in the spatial regions that intersect the two VECSEL modes. Moreover, to measure the correlations between the pump regions that pump the two modes, we must record their noises simultaneously. To this aim we built the experiment setup schematized in Fig. 3. The laser diode (LD) and the lens tube are the ones used to pump the DFVCSEL. A beam splitter follows the lens tube to split the pump beam into two identical arms. At the focal point, two pinholes of $50 \mu \mathrm{m}$ radii are placed to mimic the two laser modes. Each of the pinholes is controlled by a three dimension translation stage. We can thus record the two pump noises as a function of their separation $d$ on the structure. After passing through the pinholes, the lens and the neutral density filters, the beams are sent to PDs with a photosensitive area of $2.4 \mathrm{~mm} \times 2.8 \mathrm{~mm}$ and a bandwidth of $30 \mathrm{MHz}$. The photocurrent signals are separated into DC and AC signals by a bias-tee. The AC signals are amplified by RFAs. The two signals are sampled by a digital oscilloscope simultaneously.

Fig. 4 shows the relative intensity noise (RIN) of the pump. RIN is defined as $R I N=\left\langle|\delta \tilde{P}(f)|^{2}\right\rangle / P_{0}^{2}$, where $\delta \tilde{P}(f)$ is the Fourier Transform of the fluctuation of $P(t),\langle\rangle$ denotes the ensemble average, $P_{0}$ is the average of $P(t)$. Here, $P$ is the pump power. For simplicity, in subsequent modeling, we take the RIN of the pump to be white in the range from $10 \mathrm{kHz}$ to $30 \mathrm{MHz}$, and take it equal to $-130 \mathrm{~dB} / \mathrm{Hz}$.

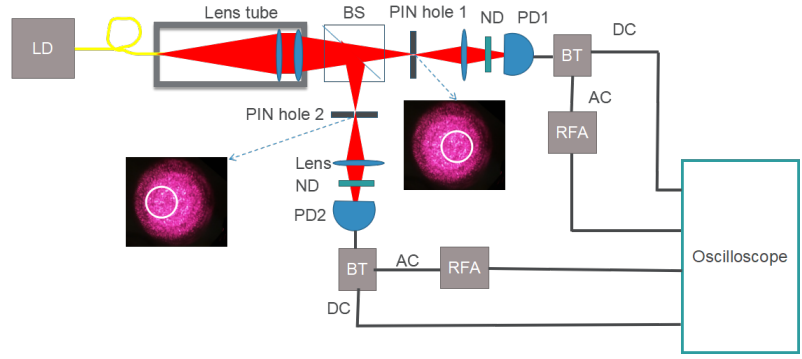

Fig. 3. Experimental setup for pump noise measurement

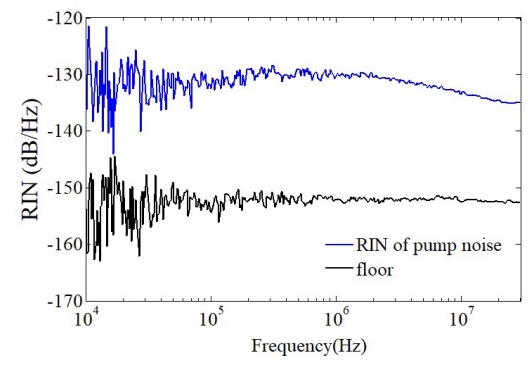

Fig. 4. RIN spectrum of the pump laser
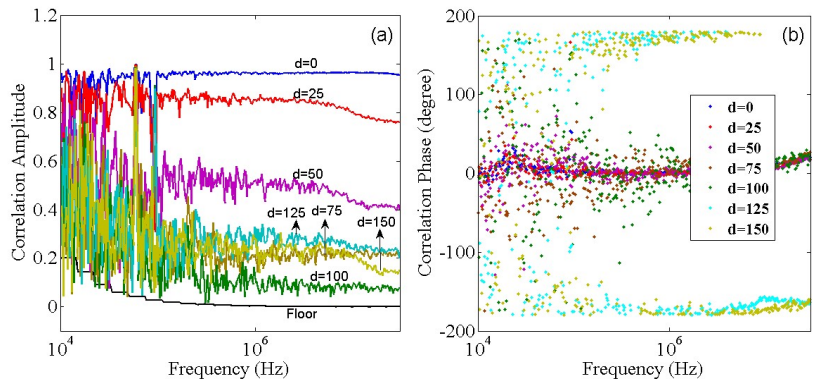

Fig. 5. (a) Amplitude and (b) phase of the correlation between the pump noise in two regions separated by distance $d$.

Fig. 5 shows the results of the correlations between the two pump noises selected by pinholes of $50 \mu \mathrm{m}$ radius whose 
images are separated by a distance $d$ in the transverse plane. The measurement starts with $d=0$, for which the correlation amplitude is almost equal to 1, illustrating the identity of the two pump regions passing through the pinholes. When $d$ increase from 0 to $100 \mu \mathrm{m}$, the correlation amplitude decreases, and the correlation phase concentrates around zero. However, when $d$ increase up to $125 \mu \mathrm{m}$ and $150 \mu \mathrm{m}$, the correlation amplitude increase again, and the correlation phase tends to $\pm 180^{\circ}$.

\section{B. Intensity noise measurement and modeling}

The intensity and phase noises of the DF-VECSEL are measured by the experimental setup shown in Fig. 6. The beam emitted by the DF-VECSEL is split into two arms. In order to measure the intensity noise of the ordinary (o) and the extraordinary (e) modes separately, the orthogonal polarizations in one arm are separated by using a half wave plate and a PBS. The other arm, projecting the o and e modes into a same polarization direction, is used for the detection of the beatnote. Then, the three beams are sent into three PDs with a $1 \mathrm{GHz}$ bandwidth. After amplification by RFAs, the intensity noise and beatnote are recorded by a deep memory digital oscilloscope.

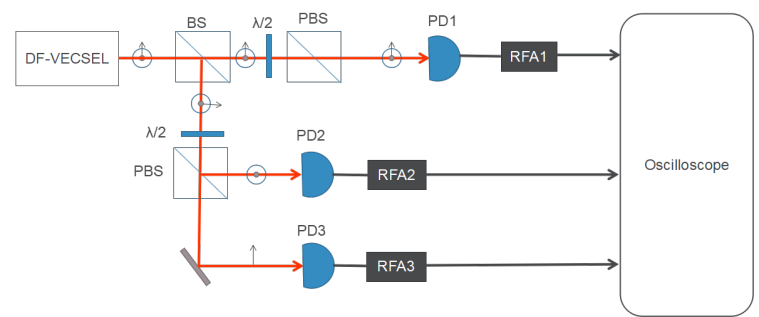

Fig. 6. Schematic of the experimental setup for intensity and phase noise measurement.

The modeling is based on modified laser rate equations. According to this theory, the Fourier transforms of the intensity noises of the $x$ - and $y$-polarized modes can be related in the frequency domain to the pump noises by a matrix [9], which is

$$
\left[\begin{array}{l}
\delta \widetilde{F}_{x}(f) \\
\delta \widetilde{F}_{y}(f)
\end{array}\right]=\left[\begin{array}{cc}
M_{x x}(f) & M_{x y}(f) \\
M_{y x}(f) & M_{y y}(f)
\end{array}\right]\left[\begin{array}{c}
\delta \widetilde{N}_{0 x}(f) \\
\delta \widetilde{N}_{0 y}(f)
\end{array}\right]
$$

with

$$
\begin{gathered}
M_{x x}=\left[1 / \tau_{y}-2 i \pi f\left(r_{y} / \tau-2 i \pi f\right) / \kappa F_{y 0}\right] / \Delta(f) \tau, \\
M_{y y}=\left[1 / \tau_{x}-2 i \pi f\left(r_{x} / \tau-2 i \pi f\right) / \kappa F_{x 0}\right] / \Delta(f) \tau, \\
M_{x y}=-\xi_{x y} / \tau \tau_{x} \Delta(f), \\
M_{y x}=-\xi_{y x} / \tau \tau_{y} \Delta(f),
\end{gathered}
$$

where

$$
\begin{aligned}
\Delta(f)= & {\left[1 / \tau_{x}-2 i \pi f\left(r_{x} / \tau-2 i \pi f\right) / \kappa F_{x 0}\right] } \\
& {\left[1 / \tau_{y}-2 i \pi f\left(r_{y} / \tau-2 i \pi f\right) / \kappa F_{y 0}\right]-C / \tau_{x} \tau_{y} }
\end{aligned}
$$

In these equations, $\delta \widetilde{F}_{x}, \delta \widetilde{F}_{y}$ denote the Fourier transforms of fluctuations in photon numbers for the two modes inside the laser cavity. $F_{x 0}$ and $F_{y 0}$ are the average photon numbers. $\delta \tilde{N}_{0 x}$ and $\delta \tilde{N}_{0 y}$ denote the pump noise spectra for the two modes, $\tau$ is the population inversion lifetime, $\tau_{x}, \tau_{y}$ are the photon lifetimes for the two modes, $r_{x}, r_{y}$ denote their excitation ratios, $\xi_{x y}, \xi_{y x}$ denote the cross- to self-saturation coefficient ratios, $C=\xi_{x y} \xi_{y x}$, and $\kappa$ is proportional to the stimulated emission cross section.
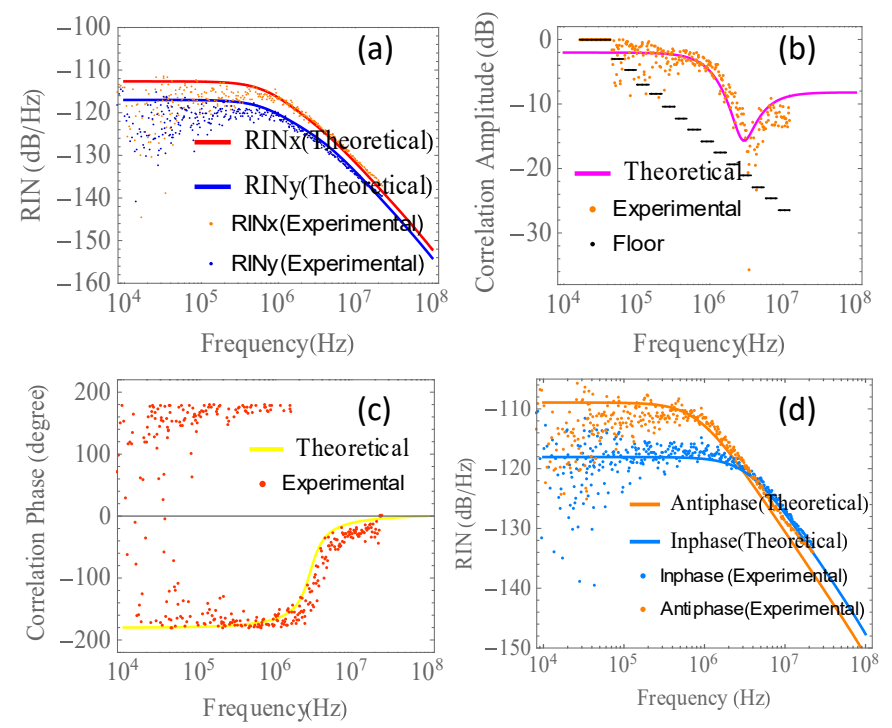

Fig. 7. The results of intensity noise measurement and modeling. Dotted lines denote the experimental results. (a) is the RIN of the intensity noise, (b) is the correlation amplitude, (c) is the correlation phase, (d) is the RIN of inphase and anti-phase noise. The parameters used in the modeling are $\tau=1 \mathrm{~ns}$, $\tau_{x}=15 n s, \tau_{y}=19 n s, r_{x}=1.53, r_{y}=1.59, R_{\text {pump }}=-132 \mathrm{~dB} / \mathrm{Hz}$, correlation amplitude $\eta=0.38$ and phase $\psi=0, C=0.38$.

Figure 7 shows the results of intensity noise measurement and modeling. Fig. 7 (a) is the RIN spectrum for the two modes. The shape of the RIN spectrum exhibits the typical first-order filter shape for class-A laser dynamics. Figures 7 (b) and 7 (c) are the amplitude and phase of the intensity noise correlation spectrum between the two modes. As can be seen, the correlation amplitude is flat and strong at the lower frequencies, before dropping and exhibiting a minimum in a narrow frequency band, after which it remains flat at a relatively low value. The corresponding correlation phase changes from about $\pm 180^{\circ}$ to $0^{\circ}$. This spectral behavior can be explained by considering the DF-VECSEL as a coupled oscillator system. Then, this system has two noise eigenmodes, i. e. in-phase mode (sum of the intensities of the two laser modes) and antiphase mode (difference of the intensities of the two modes). As an approximation, supposing the two laser modes have the same gain and losses, the in-phase and anti-phase noise complex spectra are defined as $\delta \widetilde{F}_{I n}=\delta \widetilde{F}_{x}+\delta \widetilde{F}_{y}$, $\delta \widetilde{F}_{A n t i}=\delta \widetilde{F}_{x}-\delta \widetilde{F}_{y}$. The RIN spectra of the in-phase and antiphase noises are shown in the Fig. 7 (d). One notices that the anti-phase noise is dominant over the in-phase noise at low frequencies, and conversely at higher frequencies. Since the correlation between $\delta \widetilde{F}_{x}$ and $\delta \widetilde{F}_{y}$ reads

$$
\begin{aligned}
& \operatorname{Cor}_{\delta \widetilde{F}_{x}, \delta \widetilde{F}_{y}}=\frac{\left\langle\delta \widetilde{F}_{x} \cdot \delta \widetilde{F}_{y}{ }^{*}\right\rangle}{\sqrt{\left\langle\left.\delta \widetilde{F}_{x}\right|^{2}\right\rangle \cdot\left\langle\left|\delta \widetilde{F}_{y}\right|^{2}\right\rangle}}= \\
& =\frac{\left\langle\left.\delta \widetilde{F}_{I n}\right|^{2}-\left|\delta \widetilde{F}_{A n t i}\right|^{2}+\left(\delta \widetilde{F}_{A n t i} \cdot \delta \widetilde{F}_{I n}{ }^{*}-\delta \widetilde{F}_{I n} \cdot \delta \widetilde{F}_{A n t i}{ }^{*}\right)\right\rangle}{4 \sqrt{\left\langle\left.\delta \widetilde{F}_{x}\right|^{2}\right\rangle \cdot\left\langle\left|\delta \widetilde{F}_{y}\right|^{2}\right\rangle}} \\
& =\frac{\left\langle\left|\delta F_{\text {In }}\right|^{2}-\left|\delta F_{A n t i}\right|^{2}\right\rangle}{4 \sqrt{\left\langle\left|\delta \widetilde{F}_{x}\right|^{2}\right\rangle \cdot\left\langle\left|\delta \widetilde{F}_{y}\right|^{2}\right\rangle}}
\end{aligned}
$$


the spectral behavior of the correlation between $\delta F_{x}$ and $\delta F_{y}$ can be readily explained. Since the difference between inphase and anti-phase intensity noise is large at low frequency, the correlation amplitude is high. However, when the difference between the amplitudes of the two mechanisms becomes smaller, the correlation amplitude decreeases. Besides, the correlation phase tends to be $\pm 180^{\circ}$ when the anti-phase noise is dominant, and $0^{\circ}$ when the in-phase noise is dominant. At the crossing point of the in-phase and anti-phase noise mechanisms, a dip appears due to the lower correlation amplitude.

\section{Phase noise of beatnote measurement and modeling}

Figure 8 shows the phase noise measurement and modeling. The phase noise spectrum clearly exhibits a change of slope at around $300 \mathrm{kHz}$, showing that two mechanisms have to be considered, namely the one related to thermal effects and the one attributed to Henry's phase/amplitude coupling [10]. Considering the two effects, the phase noise can be expressed as [10]:

$$
\begin{gathered}
\delta \widetilde{\phi}_{\text {Beat }}(f)=\delta \widetilde{\phi}_{H}(f)+\delta \widetilde{\phi}_{T}(f) \\
\delta \widetilde{\phi}_{H}(f)=\frac{\alpha}{2}\left(\frac{\delta \widetilde{F}_{x}(f)}{F_{x 0}}-\frac{\delta \widetilde{F}_{y}(f)}{F_{y 0}}\right) \\
\delta \widetilde{\phi}_{T}(f)=\frac{\omega_{0}}{i 2 \pi f} \Gamma_{T} R_{T} \frac{1}{1+i 2 \pi f \tau_{T}} P_{P}\left(\frac{\delta N_{0 x}(f)}{N_{0 x}}-\frac{\delta N_{0 y}(f)}{N_{0 y}}\right)
\end{gathered}
$$

where $\alpha$ is Henry's factor, $\omega_{0}$ is the frequency of laser, $R_{T}$ is the thermal resistance, $P_{p}$ is the pump power. $\Gamma_{T}$ is defined as

$$
\Gamma_{T}=\left(L_{s c} / L_{e x t}\right)(d n / d T)
$$

where $L_{S C}$ and $L_{e x t}$ are the lengths of the semiconductor structure and the external cavity, respectively, $d n / d T$ is the average thermal coefficient of the structure refractive index. $\tau_{T}$ is the thermal response time given by

$$
\tau_{T} \approx w_{p}^{2} / 2 \pi D_{T}
$$

Where $w_{p}$ is the waist of pump beam and $D_{T}$ is the thermal diffusion coefficient.

Based on these equations, the modeling is in very good agreement with the experimental result.

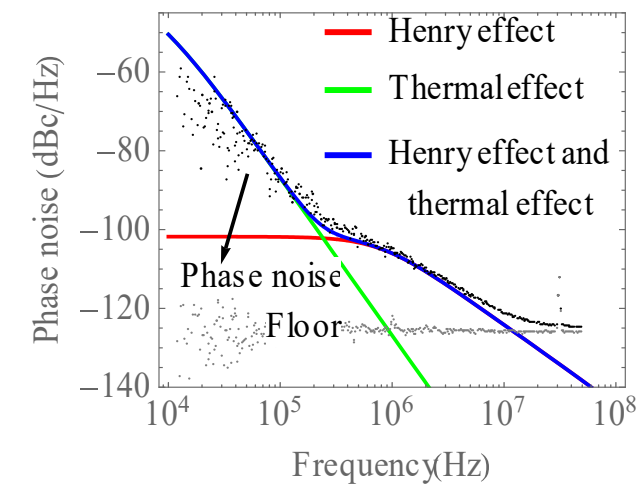

Fig. 8. Results of phase noise of the beatnote measurement and modeling. Dotted lines denote the experimental results. Parameters used in the modeling are $\alpha=3, \tau_{\mathrm{T}}=1.4 \times 10^{-5} \mathrm{~s}, \mathrm{R}_{\mathrm{T}}=6.7 \mathrm{KW}^{-1}, \Gamma_{\mathrm{T}}=3.87 \times 10^{-8} \mathrm{~K}^{-1} \mathrm{P}_{\mathrm{p}}=1.1 \mathrm{~W}$.

\section{CONCLUSIONS}

In this paper, we have studied the noise mechanisms in a DF-VECSEL operating at the wavelength of Cesium clocks. We observed the beatnote sitting on a noise pedestal of a few tens of $\mathrm{MHz}$ bandwidth, and analyzed its origin. Firstly, the pump noise, including RIN and correlation between the two pump noises at different spatial locations, is measured in a dedicated setup. Since the pump noise is the major source of intensity noise and phase noise of the beatnote, this measurement allows us to model the laser noise accordingly. Secondly, the intensity noise was measured, including the RIN and the correlation beteween the noises of the two modes. The results was well predicted by a modified rate equation model. The behavior of the correlation spectra was explained by the existence and response of in-phase and anti-phase noise mechanisms. Finally, the phase noise of the beatnote was measured and modeled by considering the effect of phase/amplitude coupling in the quantum well and thermal effect of the semiconductor chip induced by the pump laser. The agreement between the experimental and theoretical results gives us a good understanding of the noise behavior of this laser.

\section{ACKNOWLEDGMENT}

The authors thank G. Pillet for providing the computer codes for computing the noise spectra and S. De for his help. This work was supported by the Agence Nationale de la Recherche and the Direction Générale de l'Armement. The work of HL, FB, FG and GB is performed in the framekwork of the joint lab between laboratoire Aimé Cotton and Thales Research \& Technology.

\section{REFERENCES}

[1] J. Capmany, and D. Novak. "Microwave Photonics Combines Two Worlds." Nature Photonics 1, no. 6 (2007): 319-30.

[2] G. Baili, L. Morvan, M. Alouini, D. Dolfi, F. Bretenaker, I. Sagnes, and A. Garnache. "Experimental Demonstration of a Tunable DualFrequency Semiconductor Laser Free of Relaxation Oscillations." Opt Lett 34, no. 21 (Nov 01 2009): 3421-3.

[3] F.T. Arecchi, G. L. Lippi, G. P. Puccioni, and J. R. Tredicce. "Deterministic Chaos in Laser with Injected Signal." Optics Communications 51, no. 5 (1984): 308-14.

[4] J. Vanier, "Atomic Clocks Based on Coherent Population Trapping: A Review." Applied Physics B 81, no. 4 (2005): 421-42.

[5] T. Zanon, S. Guerandel, E. de Clercq, D. Holleville, N. Dimarcq, and A. Clairon. "High Contrast Ramsey Fringes with Coherent-PopulationTrapping Pulses in a Double Lambda Atomic System." Phys Rev Lett 94, no. 19 (May 20 2005): 193002.

[6] P. Dumont, F. Camargo, J.M. Danet, D. Holleville, S. Guerandel, G. Pillet, G. Baili, et al. "Low-Noise Dual-Frequency Laser for Compact Cs Atomic Clocks." Journal of Lightwave Technology 32, no. 20 (2014): 3817-23.

[7] M. Brunel, F. Bretenaker, and A. Le Floch. "Tunable Optical Microwave Source Using Spatially Resolved Laser Eigenstates." Optics Letters 22, no. 6 (1997): 384 .

[8] S. De, G. Baili, M. Alouini, J. C. Harmand, S. Bouchoule, and F. Bretenaker. "Class-a Dual-Frequency Vecsel at Telecom Wavelength." Opt Lett 39, no. 19 (Oct 01 2014): 5586-9.

[9] S. De, V. Pal, A. El Amili, G. Pillet, G. Baili, M. Alouini, I. Sagnes, R. Ghosh, and F. Bretenaker. "Intensity Noise Correlations in a TwoFrequency Vecsel." Opt Express 21, no. 3 (Feb 11 2013): 2538-50.

[10] S. De, A. E. Amili, I. Fsaifes, G. Pillet, G. Baili, F. Goldfarb, M. Alouini, I. Sagnes, and F. Bretenaker. "Phase Noise of the Radio Frequency (Rf) Beatnote Generated by a Dual-Frequency Vecsel." Journal of Lightwave Technology 32, no. 7 (2014): 1307-16.

[11] S. De, G. Baili, S. Bouchoule, M. Alouini, and F. Bretenaker. "Intensity- and Phase-Noise Correlations in a Dual-Frequency VerticalExternal-Cavity Surface-Emitting Laser Operating at Telecom Wavelength." Physical Review A 91, no. 5 (2015). 